\title{
EXPONENTIAL ATTRACTORS \\ FOR SEMILINEAR WAVE EQUATIONS WITH DAMPING
}

\author{
ALBERT MILANI \\ Department of Mathematical Sciences, University of Wisconsin-Milwaukee \\ P.O. Box 413, Milwaukee, Wisconsin 53201, U.S.A.
}

\section{Introduction}

1. In this lecture we propose to present some results on the existence of exponential attractors for the semilinear damped wave equation

$$
u_{t t}+u_{t}-\Delta u+g(u)=f
$$

that we have recently obtained in cooperation with Dr. Alp Eden of Arizona State University; these results are contained in [4] and [5]. Typical examples of nonlinearities we consider for (1) are

$$
\begin{aligned}
& g(u)=\sin u, \quad \text { in any space dimension, } \\
& g(u)=u^{3}+p(u), \quad p \text { quadratic, } \quad \text { in three space dimensions . }
\end{aligned}
$$

Our goal is to describe the long time behavior of solutions to (1), by means of a set, called exponential attractor or inertial set, which is finite-dimensional, contains the global attractor and attracts the solutions of (1) at an exponential rate.

2. The recently developed theory of exponential attractors has revealed itself to be an extremely powerful tool for the description of the asymptotic behavior of infinite-dimensional dynamical systems; it retains many aspects of both the theories of global attractors and inertial manifolds (refer e.g. to Hale [6] or Temam [8]), while requiring, so to speak, less stringent conditions for its construction.

The main difference between exponential attractors and global attractors lies in the fact that all solutions converge to the exponential attractor at a uniform exponential rate, once they are in an absorbing ball. Thus, the exponential attractor contains the global attractor, and those stable manifolds where the rate of convergence is only polynomial. However, in contrast to inertial manifolds, which also have finite dimension and attract solutions exponentially, exponential attractors are not required to have a manifold structure; indeed, a simple way of 
constructing an exponential attractor would be to restrict the inertial manifold to an absorbing set.

3. We briefly recall the main definitions concerning exponential attractors. Let $X$ be a separable Hilbert space on which a dynamical system, typically arising from an initial value problem for a dissipative differential equation such as (1), is described by a solution operator $S(t): X \rightarrow X, S$ being a continuous semigroup. The first step consists in showing the existence of a bounded absorbing set for $S(t)$ (usually a ball), that is, a bounded invariant set $B \subseteq X$ into which all solutions eventually enter; namely,

(i) $\forall t \geq 0, \quad S(t) B \subseteq B$,

(ii) $\forall u_{0} \in X \exists t_{0}=t_{0}\left(\left\|u_{0}\right\|_{X}\right) \forall t \geq t_{0}, \quad S(t) u_{0} \in B$.

4. The next step consists in showing that the $\omega$-limit set of $B$ is the global attractor of $S(t)$; namely, if

$$
A=\omega(B) \equiv \bigcap_{s \geq 0} \overline{\bigcup_{t \geq s} S(t) B}
$$

then $A$ is compact, invariant, finite-dimensional and attracts all solutions, i.e.

$$
\forall u_{0} \in X, \quad \lim _{t \rightarrow+\infty} d_{X}\left(S(t) u_{0}, A\right)=0 .
$$

Although the global attractor completely describes the asymptotic behavior of the dynamical system, it is in general quite difficult, in concrete examples, to describe its geometrical and differential structure; also, the rate of convergence of solutions in (3) may be quite slow and, finally, the available estimates on the dimension of the attractor may be extremely rough.

5. To counter these problems, one looks for the existence of inertial manifolds, that is, of sets which are Lipschitz manifolds of finite dimension, flow invariant and exponentially attracting. Namely, $X$ is decomposed into an $N$-dimensional component $P_{N} X$ and its orthogonal complement $Q_{N} X$, and a Lipschitz function $\phi: P_{N} X \rightarrow Q_{N} X$ is sought such that if $M$ is its graph, then

(i) $S(t) M \subseteq M$,

(ii) $\forall u_{0} \in B \exists c_{0}, c_{1}>0 \exists t_{0} \forall t \geq t_{0}, \quad d_{X}\left(S(t) u_{0}, M\right) \leq c_{1} e^{-c_{0} t}$.

If such a $\phi$ exists, $M$ is called an inertial manifold; it has finite dimension not greater than $N$, and $A \subseteq M \cap B$. In particular, the decomposition

$$
u=P_{N} u+\phi\left(P_{N} u\right)
$$

shows that the evolution of the system can be described by a finite number of ordinary differential equations. Clearly, the geometrical and differential structure of $M$ is completely described by $\phi$; at present, however, the existing theory of inertial manifolds hinges heavily on a condition on the growth of the eigenvalues $\left\{\lambda_{n}\right\}$ of the operator in the evolution equation, called the gap condition. This is 
a quite strong condition on the divergence of the difference $\lambda_{n+1}-\lambda_{n}$ as $n \rightarrow \infty$, and is extremely difficult to verify in concrete examples (for instance, it is not known if it holds for the 2-dimensional Navier-Stokes equations). Moreover, here again the available estimates on the dimension of the inertial manifolds are too crude.

6. To counter this other set of problems, it is expedient to introduce a smaller set $Z$, the exponential attractor. This set is compact and contains the attractor; like the inertial manifold, it is finite-dimensional, flow invariant and attracts solutions exponentially, but with a uniform rate. Namely,

(i) $S(t) Z \subseteq Z$,

(ii) $\exists c_{0}, c_{1}>0 \forall u_{0} \in B \forall t \geq 0, \quad d_{X}\left(S(t) u_{0}, Z\right) \leq c_{1} e^{-c_{0} t}$.

We remark that in general, when all these sets exist, they are related by the following set inclusions:

$$
A \subseteq Z \subseteq M \cap B \subseteq B .
$$

7. As we have mentioned, whenever an inertial manifold $M$ exists, the set $Z=M \cap B$ is an exponential attractor; however, in light of the problems described above, it is expedient to resort to a different approach to construct the exponential attractors directly. This can be obtained by adding to the global attractor a certain set of points that fail to satisfy a condition, called the discrete squeezing property. One way of describing this property is the following:

Definition. The solution operator $S(t)$ satisfies the discrete squeezing property on $B$ if there exist $t_{*}>0$ and an orthogonal projection $P$ of finite rank $N_{0}$ such that, $\forall u, \forall v \in B$, either

$$
\left\|S_{*} u-S_{*} v\right\|_{X} \leq \frac{1}{8}\|u-v\|_{X},
$$

where $S_{*}=S\left(t_{*}\right)$, or

$$
\left\|(I-P)\left(S_{*} u-S_{*} v\right)\right\|_{X} \leq\left\|P\left(S_{*} u-S_{*} v\right)\right\|_{X} .
$$

In other words, if the infinite-dimensional part dominates the finite one, the map $S_{*}$ is actually a contraction or, to describe the property in another way, if $w_{*}=$ $S_{*} u-S_{*} v$,

$$
\left\|w_{*}\right\|_{X}>\sqrt{2}\left\|P w_{*}\right\|_{X} \Rightarrow\left\|w_{*}\right\|_{X} \leq \frac{1}{8}\|u-v\|_{X} .
$$

8. The importance of the discrete squeezing property for the construction of exponential attractors is based on the following

THEOREM 1. If the solution operator $S(t)$ satisfies the discrete squeezing property on a bounded absorbing set $B$, then there exists an exponential attractor $Z \subseteq B$ satisfying (5) and whose (fractal) dimension is of the order of $N_{0}$.

Proof. See Eden, Foiaş, Nicolaenko and Temam [3].

Our goal is thus to show that the solution operator associated with equation (1) does satisfy the discrete squeezing property and, therefore, possesses an 
exponential attractor. Before this, however, we conclude this introduction by recalling several models of equations for which exponential attractors have been shown to exist in this way; namely, the 2-dimensional Navier-Stokes equations, the Kuramoto-Sivashinsky equations with periodic boundary conditions

$$
u_{t}+u_{x x x x}+u_{x x}+u u_{x}=f,
$$

the Chaffee-Infante equations with Dirichlet boundary conditions

$$
u_{t}-\Delta u+u^{3}-u=f \text {, }
$$

and the original Burger's equations

$$
\left\{\begin{array}{l}
U_{t}=R-U-\int_{0}^{1}|v|^{2} d x \\
v_{t}=U v+v_{x x}-\left(v^{2}\right)_{x}
\end{array}\right.
$$

(see Eden-Foiaş-Nicolaenko-Temam [3] and Eden [2]).

\section{Statement of results}

1. We now turn to a concrete example of equation (1), concentrating on the quantum mechanics nonlinearity $g(u)=u^{3}-u$. Let $\Omega \subseteq \mathbb{R}^{3}$ be a bounded open domain with a smooth boundary $\partial \Omega$; we consider the semilinear initial-boundary value problem

$$
\left\{\begin{array}{l}
\varepsilon u_{t t}+u_{t}-\Delta u+u^{3}-u=f(x, t), \\
u(x, 0)=u_{0}(x), \quad u_{t}(x, 0)=u_{1}(x),\left.\quad u\right|_{\partial \Omega}=0,
\end{array}\right.
$$

where $\varepsilon>0$. We consider (2.1) as an evolution equation for the pair $\left\{u(t), u_{t}(t)\right\}$ $=S^{\varepsilon}(t)\left\{u_{0}, u_{1}\right\}$, on the Hilbert space $E_{0}=V \times H, V=H_{0}^{1}(\Omega), H=L^{2}(\Omega)$; setting also $Y=H^{2}(\Omega) \cap H_{0}^{1}(\Omega)$ and $E_{1}=Y \times V$, the existence of the solution operator, and its regularity properties, are assured for all $\varepsilon>0$ by the following

Theorem 2. (i) $\forall f \in C_{\mathrm{b}}\left(\mathbb{R}^{+} ; H\right) \forall\left\{u_{0}, u_{1}\right\} \in E_{0} \exists ! u \in C_{\mathrm{b}}\left(\mathbb{R}^{+} ; V\right) \cap C_{\mathrm{b}}^{1}\left(\mathbb{R}^{+} ; H\right)$, solution of (2.1).

(ii) If moreover $f \in C_{\mathrm{b}}^{1}\left(\mathbb{R}^{+} ; H\right)$ and $\left\{u_{0}, u_{1}\right\} \in E_{1}$, then $u \in C_{\mathrm{b}}\left(\mathbb{R}^{+} ; Y\right) \cap$ $C_{\mathrm{b}}^{1}\left(\mathbb{R}^{+} ; V\right) \cap C_{\mathrm{b}}^{2}\left(\mathbb{R}^{+} ; H\right)$

Proof. See Temam [8] or Babin-Vishik [1].

The existence of attractors and inertial manifolds for $S^{\varepsilon}(t)$, when $f$ is independent of $t$, is described by

THEOREM 3. (i) $\forall \varepsilon>0$, there exists a compact attractor $A_{\varepsilon}$ for $S^{\varepsilon}(t)$ in $E_{0}$.

(ii) If $\varepsilon \gg 1$, there exists no $C^{1}$ inertial manifold for $S^{\varepsilon}(t)$.

(iii) If $\varepsilon \ll 1$, there exists a $C^{1}$ inertial manifold for $S^{\varepsilon}(t)$, at least in one space dimension.

Proof. For (i), see Babin-Vishik [1] and also Eden-Milani [4] if $\varepsilon$ is small; for (ii) and (iii), see Mora-Solà-Morales [7]. 
We now come to our result on the existence of exponential attractors for equation (2.1):

TheOrem 4. Assume $f \in C_{\mathrm{b}}^{1}\left(\mathbb{R}^{+} ; H\right)$. Then $\forall \varepsilon>0, S^{\varepsilon}(t)$ admits an absorbing ball $B_{1} \subseteq E_{1}$ over which it satisfies the discrete squeezing property.

Proof. See the next section.

2. We consider the following norms in $E_{0}$ and $E_{1}$ :

$$
\|\{u, v\}\|_{E_{0}}^{2}=\varepsilon|v|^{2}+|\nabla u|^{2}, \quad\|\{u, v\}\|_{E_{1}}^{2}=\varepsilon|\nabla v|^{2}+|\Delta u|^{2},
$$

where $|\cdot|$ is the norm in $L^{2}(\Omega)$; we recall that these norms are equivalent to the standard ones, because of Poincaré's inequalities; in particular,

$$
\exists p>0 \forall u \in Y, \quad|\nabla u|^{2} \leq p|\Delta u|^{2} .
$$

For the sake of simplicity, we limit ourselves to the case $\varepsilon \leq 1$; defining then, for $w=\{u, v\} \in E_{1}$, the function

$$
N_{1}(w)=\varepsilon|\nabla v|^{2}+\varepsilon(\nabla u, \nabla v)+\frac{1}{2}|\nabla u|^{2}+|\Delta u|^{2}+3\left(u^{2} \nabla u, \nabla u\right),
$$

a simple application of Schwarz' inequality yields the inequality

$$
\forall\{u, v\}=w \in E_{1}, \quad\|w\|_{E_{1}}^{2} \leq 2 N_{1}(w) .
$$

We can now start the proof of Theorem 4. After Temam [8] we can assume the existence of an absorbing ball $B_{0} \subseteq E_{0}$ for $S^{\varepsilon}(t)$, that is, if $\left\{u(t), u_{t}(t)\right\}=$ $S^{\varepsilon}(t)\left\{u_{0}, u_{1}\right\}$,

(2.4) $\exists R_{0}>0 \exists T_{0}=T\left(R_{0}\right) \forall t \geq T_{0} \forall\left\{u_{0}, u_{1}\right\} \in E_{0}, \quad \varepsilon\left|u_{t}(t)\right|^{2}+|\nabla u(t)|^{2} \leq R_{0}^{2}$.

We claim then

Proposition 1. $\exists R_{1}>0 \exists T_{1}=T\left(R_{1}, T_{0}, R_{0}\right) \geq T_{0} \forall t \geq T_{1} \forall\left\{u_{0}, u_{1}\right\} \in E_{1}$,

$$
\varepsilon\left|\nabla u_{t}(t)\right|^{2}+|\Delta u(t)|^{2} \leq R_{1}^{2} \text {. }
$$

Pr o of. We multiply the equation of (2.1) in $H$ by $-\Delta u$ and $-2 \Delta u_{t}$ to obtain

$$
\begin{aligned}
& \frac{d}{d t}\left\{\varepsilon\left|\nabla u_{t}\right|^{2}+\varepsilon\left(\nabla u, \nabla u_{t}\right)+\frac{1}{2}|\nabla u|^{2}+|\Delta u|^{2}+3\left(u^{2} \nabla u, \nabla u\right)+2(f, \Delta u)\right\} \\
& \quad+(2-\varepsilon)\left|\nabla u_{t}\right|^{2}+|\Delta u|^{2}+3\left(u^{2} \nabla u, \nabla u\right)+2 \mu(f, \Delta u) \\
& =(2 \mu-1)(f, \Delta u)+2\left(f_{t}, \Delta u\right)+6(u \nabla u, \nabla u)+2\left(\nabla u, \nabla u_{t}\right)+|\nabla u|^{2} \equiv r,
\end{aligned}
$$

where $\mu=\min [1 / 3,1 /(2(p+1))]$, with $p$ defined in $(2.2)$.

Recalling then (2.4), we estimate $r$ for $t \geq T_{0}$ as follows:

$$
\begin{aligned}
r \leq & \frac{3}{2}|2 \mu-1||f|^{2}+\frac{1}{6}|\Delta u|^{2}+6\left|f_{t}\right|^{2}+\frac{1}{6}|\Delta u|^{2} \\
& \quad+36|\nabla u|^{4}+\frac{1}{6}|\Delta u|^{2}+2|\nabla u|^{2}+\frac{1}{2}\left|\nabla u_{t}\right|^{2}+|\nabla u|^{2} \\
\leq & \frac{3}{2}|f|^{2}+6\left|f_{t}\right|^{2}+36 R_{0}^{4}+3 R_{0}^{2}+\frac{1}{2}|\Delta u|^{2}+\frac{1}{2}\left|\nabla u_{t}\right|^{2} ;
\end{aligned}
$$


thus, there exists a constant $\gamma_{1}>0$, depending only on $R_{0}$ and the norm of $f$ in $C_{\mathrm{b}}^{1}\left(\mathbb{R}^{+} ; H\right)$, such that, if $t \geq T_{0}$,

$$
\begin{aligned}
\frac{d}{d t}\left\{N_{1}(u, t)+2(f, \Delta u)\right\}+\frac{3}{2} \varepsilon\left|\nabla u_{t}\right|^{2} & +\frac{1}{2}|\Delta u|^{2} \\
& +3\left(u^{2} \nabla u, \nabla u\right)+2 \mu(f, \Delta u) \leq \gamma_{1} .
\end{aligned}
$$

It is now easy to see that, if $\varepsilon \leq 1$,

$$
\mu\left\{N_{1}(u, t)+2(f, \Delta u)\right\} \leq \frac{3}{2} \varepsilon\left|\nabla u_{t}\right|^{2}+\frac{1}{2}|\Delta u|^{2}+3\left(u^{2} \nabla u, \nabla u\right)+2 \mu(f, \Delta u),
$$

so that (2.6) implies

$$
\frac{d}{d t}\left\{N_{1}(u, t)+2(f, \Delta u)\right\}+\mu\left\{N_{1}(u, t)+2(f, \Delta u)\right\} \leq \gamma_{1}
$$

from which we deduce that for $t \geq T_{0}$

(2.7) $\left\{N_{1}\left(u, u_{t}\right)+2(f, \Delta u)\right\}(t) \leq e^{-\mu\left(t-T_{0}\right)}\left\{N_{1}\left(u, u_{t}\right)+2(f, \Delta u)\right\}\left(T_{0}\right)+\gamma_{1} \mu^{-1}$.

From (ii) of Theorem 2 we know that there exists $\gamma_{2}>0$, depending on the norm of $\left\{u_{0}, u_{1}\right\}$ in $E_{1}$, and on that of $f$ on $C_{\mathrm{b}}^{1}\left(\mathbb{R}^{+} ; H\right)$, such that

$$
\left\{N_{1}\left(u, u_{t}\right)+2(f, \Delta u)\right\}\left(T_{0}\right) \leq \gamma_{2}
$$

from (2.7) we then obtain

$$
\left\{N_{1}\left(u, u_{t}\right)+2(f, \Delta u)\right\}(t) \leq 2 \gamma_{1} \mu^{-1}
$$

for $t \geq T_{1}$, where $T_{1}=T_{0}+(1 / \mu) \ln \left(\gamma_{2} \mu / \gamma_{1}\right)\left(\geq T_{0}\right)$.

We now recall (2.3) to deduce that for $t \geq T_{1}$, (2.8) yields

$\left\|\left\{u, u_{t}\right\}(t)\right\|_{E_{1}}^{2} \leq 2 N_{1}(u, t) \leq 2 \gamma_{2}-2(f, \Delta u)(t) \leq 2 \gamma_{2}+2|f(t)|^{2}+\frac{1}{2}|\Delta u(t)|^{2}$,

so that eventually we have $(2.5)$ with $R_{1}=2\left(\gamma_{2}+\|f\|_{C_{\mathrm{b}}\left(\mathbb{R}^{+} ; H\right)}\right)^{1 / 2}$

3. We now proceed to prove the discrete squeezing property for $S^{\varepsilon}(t)$. Let $\left\{\lambda_{n}\right\}$ be the sequence of the eigenvalues of $-\Delta$, and $\left\{w_{n}\right\}$ the corresponding sequence of eigenvectors. Let $H_{N}=\operatorname{span}\left\{w_{1}, \ldots, w_{N}\right\}$, and $p_{N}: H \rightarrow H_{N}$, $q_{N}=I-p_{N}$ be the corresponding orthogonal projections (which are orthogonal both in $V$ and $H$ ); clearly, we have

$$
\forall u \in q_{N} V, \quad|u|^{2} \leq \frac{1}{\lambda_{N+1}}|\nabla u|^{2} .
$$

Next, we define corresponding product projections in $E_{0}$, namely

$$
P_{N}: E_{0} \rightarrow\left(p_{N} V\right) \times\left(p_{N} H\right), \quad P_{N}(\{u, v\})=\left\{p_{N} u, p_{N} v\right\}, \quad Q_{N}=I-P_{N} .
$$

Then, for $w=\{u, v\} \in E_{0}$, we define the functions

$$
\begin{aligned}
N_{0}(w) & =\varepsilon|v|^{2}+\varepsilon(u, v)+\frac{1}{2}|u|^{2}+|\nabla u|^{2}, \\
M(w) & =\|w\|_{E_{0}}^{2}+(u, v)=\varepsilon|v|^{2}+(u, v)+|\nabla u|^{2}
\end{aligned}
$$

and claim: 
Lemma 1. (i) Let $K=\max \left\{1+1 / \lambda_{1}, 3 / 2\right\}$. Then if $\varepsilon \leq 1, N_{0}$ is an equivalent norm in $E_{0}$; indeed, $\forall w=\{u, v\} \in E_{0}$,

$$
N_{0}(w) \geq|\nabla u|^{2}, \quad \frac{1}{2}\|w\|_{E_{0}}^{2} \leq N_{0}(w) \leq K\|w\|_{E_{0}}^{2} .
$$

(ii) If $\varepsilon \leq 1$ and $N$ is so large that $\varepsilon \lambda_{N+1} \geq 1$, then $M$ is an equivalent norm in $Q_{N} E_{0}$; indeed, $\forall w \in Q_{N} E_{0}$,

$$
\|w\|_{E_{0}}^{2} \leq 2 M(w) \leq 3\|w\|_{E_{0}}^{2} .
$$

Proof. Consequence of Schwarz' inequality; in particular for (2.11), note that by (2.9) we have

$$
(u, v) \leq \frac{1}{2 \varepsilon}|u|^{2}+\frac{\varepsilon}{2}|v|^{2} \leq \frac{1}{2 \varepsilon \lambda_{N+1}}|\nabla u|^{2}+\frac{\varepsilon}{2}|v|^{2} \leq \frac{1}{2}\|w\|_{E_{0}}^{2} .
$$

We now estimate the difference of two solutions $u, \bar{u} \in C_{\mathrm{b}}\left(\mathbb{R}^{+} ; V\right) \cap C_{\mathrm{b}}^{1}\left(\mathbb{R}^{+} ; H\right)$ of (2.1): if $w=u-\bar{u}$ and $W=\left\{w, w_{t}\right\}$, so that $W \in C_{\mathrm{b}}\left(\mathbb{R}^{+} ; E_{0}\right)$, we claim

LEMma 2. Let $K$ be as in Lemma 1. There exists $\alpha>0$ such that

$$
\forall t \geq 0, \quad\|W(t)\|_{E_{0}}^{2} \leq 2 K e^{\alpha t}\|W(0)\|_{E_{0}}^{2} .
$$

Proof. $w$ solves the equation

$$
\varepsilon w_{t t}+w_{t}-\Delta w=w+u^{3}-v^{3},
$$

which we multiply by $2 w_{t}$ and $w$ to obtain

$$
\begin{aligned}
& \frac{d}{d t} N_{0}(w)+(2-\varepsilon)\left|w_{t}\right|^{2}+|\nabla w|^{2}=\left(w+u^{3}-v^{3}, 2 w_{t}+w\right) \\
& \leq\left|w_{t}\right|^{2}+\left(\frac{3}{\lambda_{1}}+18 R^{4}+\frac{3 R^{2}}{\lambda_{1}}\right)|\nabla w|^{2} \equiv\left|w_{t}\right|^{2}+\alpha|\nabla w|^{2},
\end{aligned}
$$

so that (2.12) follows by Gronwall's inequality, recalling (2.10).

LEMMA 3. Let $N$ be such that $\varepsilon \lambda_{N+1} \geq 1, q=q_{N} w, Q=Q_{N} W$. Then $\exists \beta>0$ such that

$$
\forall t \geq 0, \quad \frac{d}{d t} M(Q(t))+\frac{1}{2 \varepsilon} M(Q(t)) \leq \frac{\beta}{\lambda_{N+1}}|\nabla w(t)|^{2} .
$$

Proof. We apply $q_{N}$ to $(2.13):$ since $q_{N}$ and $-\Delta$ commute, we have

$$
\varepsilon q_{t t}+q_{t}-\Delta q=\Gamma \equiv q_{N}\left(v^{3}-u^{3}\right)+q .
$$

Multiplying this by $2 q_{t}$ and $(1 / \varepsilon) q$ we obtain

$$
\frac{d}{d t} M(Q)+\left|q_{t}\right|^{2}+\frac{1}{\varepsilon}|\nabla q|^{2}+\frac{1}{2 \varepsilon}\left(q, q_{t}\right)=-\frac{1}{2 \varepsilon}\left(q, q_{t}\right)+\left(\Gamma, 2 q_{t}+\frac{1}{\varepsilon} q\right) .
$$

Recalling (2.9), we estimate

$$
|\Gamma| \leq \frac{1}{\lambda_{N+1}^{1 / 2}}\left|\nabla\left(u^{3}-v^{3}-w\right)\right| \leq \frac{1}{\lambda_{N+1}^{1 / 2}}\left(9 R_{1}^{2}+1\right)|\nabla w|,
$$


where $R_{1}$ is the radius of the absorbing ball for $S^{\varepsilon}(t)$ in $E_{1}$, as provided by Proposition 1. Indeed, we have

$$
\begin{aligned}
\left|\nabla\left(u^{3}-v^{3}\right)\right| & \leq 3\left|\left(u^{2}-v^{2}\right) \nabla u\right|+3\left|v^{2} \nabla w\right| \\
& \leq 3\left\{\left(|u|_{L^{\infty}}+|v|_{L^{\infty}}\right)|\nabla u|_{L^{3}}|w|_{L^{6}}+|v|_{L^{\infty}}^{2}|\nabla w|\right\} \\
& \leq 3\left\{(|\Delta u|+|\Delta v|)|\Delta u||\nabla w|+|\Delta w|^{2}|\nabla w|\right\} \leq 9 R_{1}^{2}|\nabla w| .
\end{aligned}
$$

Thus, we see from (2.16) that the right side of (2.15) is estimated by

$$
\begin{aligned}
\frac{1}{2 \varepsilon}|q|\left|q_{t}\right|+\frac{1}{\lambda_{N+1}^{1 / 2}}\left(9 R_{1}^{2}+1\right) & |\nabla w|\left(2\left|q_{t}\right|+\frac{1}{\varepsilon}|q|^{2}\right) \\
\leq & \frac{1}{2}\left|q_{t}\right|^{2}+\frac{3}{8 \varepsilon^{2}}|q|^{2}+\frac{6\left(9 R_{1}^{2}+1\right)}{\lambda_{N+1}}|\nabla w|^{2} \\
\leq & \frac{1}{2}\left|q_{t}\right|^{2}+\frac{3}{8 \varepsilon} \frac{1}{\varepsilon \lambda_{N+1}}|\nabla q|^{2}+\frac{\beta}{\lambda_{N+1}}|\nabla w|^{2}
\end{aligned}
$$

with $\beta=6\left(9 R_{1}^{2}+1\right)$; inserting this in (2.15) yields (2.14).

We are now ready to show that the discrete squeezing property holds. Let $U=\left\{u_{0}, u_{1}\right\}$ and $\bar{U}_{0}=\left\{\bar{u}_{0}, \bar{u}_{1}\right\}$ be in $E_{0}$, and set $W(t)=S^{\varepsilon}(t) U-S^{\varepsilon}(t) \bar{U}$. We will show that there exist $t_{*}>0$ and $N_{0}$ such that if

$$
\left\|P_{N_{0}} W\left(t_{*}\right)\right\|_{E_{0}} \leq\left\|Q_{N_{0}} W\left(t_{*}\right)\right\|_{E_{0}}
$$

then in fact $\left\|W\left(t_{*}\right)\right\|_{E_{0}} \leq \frac{1}{8}\|W(0)\|_{E_{0}}$ as well. By Lemmas 3 and 2 we have

$$
\frac{d}{d t} M(Q)+\frac{1}{2 \varepsilon} M(Q) \leq \frac{\beta}{\lambda_{N+1}}|\nabla w|^{2} \leq \frac{\beta}{\lambda_{N+1}}\|W\|_{E_{0}}^{2} \leq \frac{2 K \beta}{\lambda_{N+1}} e^{\alpha t}\|W(0)\|_{E_{0}}^{2},
$$

from which it follows that

$$
M(Q(t)) \leq M(Q(0)) e^{-t /(2 \varepsilon)}+\frac{4 K \beta \varepsilon}{\lambda_{N+1}} e^{\alpha t}\|W(0)\|_{E_{0}}^{2}
$$

and, recalling (2.11), if $\varepsilon \lambda_{N+1} \geq 1$,

$$
\left\|Q_{N} W(t)\right\|_{E_{0}}^{2} \leq\left(9 e^{-t /(2 \varepsilon)}+\frac{4 \beta \varepsilon}{\lambda_{N+1}} e^{\alpha t}\right)\|W(0)\|_{E_{0}}^{2} .
$$

We now choose first $t_{*}$ so that $18 e^{-t_{*} /(2 \varepsilon)} \leq 1 / 128$ and then $N_{0}$ so large that $\lambda_{N_{0}+1} \geq 1 / \varepsilon$ and

$$
\frac{8 K \beta \varepsilon}{\lambda_{N+1}} e^{\alpha t_{*}} \leq \frac{1}{128}
$$

if for this choice of $t_{*}$ and $N_{0},(2.17)$ holds, then from (2.18) we deduce that

$$
\begin{aligned}
\left\|W\left(t_{*}\right)\right\|_{E_{0}}^{2} & =\left\|P_{N} W\left(t_{*}\right)\right\|_{E_{0}}^{2}+\left\|Q_{N} W\left(t_{*}\right)\right\|_{E_{0}}^{2} \leq 2\left\|Q_{N} W\left(t_{*}\right)\right\|_{E_{0}}^{2} \\
& \leq\left(18 e^{-t_{*} /(2 \varepsilon)}+8 \beta \varepsilon \lambda_{N+1}^{-1} e^{\alpha t_{*}}\right)\|W(0)\|_{E_{0}}^{2} \leq \frac{1}{64}\|W(0)\|_{E_{0}}^{2},
\end{aligned}
$$

that is, $\left\|W\left(t_{*}\right)\right\|_{E_{0}} \leq \frac{1}{8}\|W(0)\|_{E_{0}}^{2}$, as desired. The proof of Theorem 4 is thus complete; note that $t_{*}=t_{*}(\varepsilon)$ and $N_{0}=N_{0}\left(t_{*}, \varepsilon\right)$. 
Acknowledgements. The author is grateful to the organizers of the Semester in PDEs for inviting him to the Banach Center. In particular, the very kind hospitality of Prof. W. Zajączkowski was greatly appreciated.

\section{References}

[1] A. V. Babin and M. I. Vishik, Attractors of Evolution Equations, Nauka, Moscow 1989 (in Russian).

[2] A. Eden, On Burger's original mathematical model of turbulence, preprint.

[3] A. Eden, C. Foias, B. Nicolaenko et R. Temam, Ensembles inertiels pour des équations d'évolution dissipatives, C. R. Acad. Sci. Paris 310 (1990), 559-562.

[4] A. Eden and A. Milani, On the convergence of attractors and exponential attractors for singularly perturbed hyperbolic equations, preprint, 1990.

[5] A. Eden, A. Milani and B. Nicolaenko, Finite dimensional exponential attractors for semilinear wave equations with damping, to appear.

[6] J. Hale, Asymptotic Behavior of Dissipative Systems, Math. Surveys and Monographs 25, Amer. Math. Soc., Providence, R.I., 1988.

[7] X. Mora and J. Solà-Morales, Existence and non-existence of finite-dimensional globally attracting invariant manifolds in semilinear damped wave equations, in: Dynamics of Infinite Dimensional Systems, Springer, New York 1987, 187-210.

[8] R. Temam, Infinite Dimensional Dynamical Systems in Mechanics and Physics, Springer, New York 1988. 